\title{
Random Insertional Mutagenesis Identifies Genes Associated with Virulence in the Wheat Scab Fungus Fusarium graminearum
}

\author{
Kyeyong Seong, Zhanming Hou, Miles Tracy, H. Corby Kistler, and Jin-Rong Xu
}

\begin{abstract}
First, second, third, and fifth authors: Department of Botany and Plant Pathology, Purdue University, West Lafayette, IN 47907; and fourth author: U.S. Department of Agriculture-Agricultural Research Service, Cereal Disease Laboratory, Department of Plant Pathology, University of Minnesota, St. Paul 55108.
\end{abstract}

Accepted for publication 24 February 2005.

ABSTRACT

\begin{abstract}
Seong, K., Hou, Z., Tracy, M., Kistler, H. C., and Xu, J.-R. 2005. Random insertional mutagenesis identifies genes associated with virulence in the wheat scab fungus Fusarium graminearum. Phytopathology 95:744-750.

Fusarium graminearum is an important pathogen of small grains and maize in many areas of the world. To better understand the molecular mechanisms of $F$. graminearum pathogenesis, we used the restriction enzyme-mediated integration (REMI) approach to generate random insertional mutants. Eleven pathogenicity mutants were identified by screening 6,500 hygromycin-resistant transformants. Genetic analyses indicated that the defects in plant infection were tagged by the transforming vector in six of these mutants. In mutant M8, the transforming plasmid was integrated 110-bp upstream from the start codon of the cystathionine beta-
\end{abstract}

lyase gene $(C B L 1)$. Gene replacement mutants deleted for $C B L 1$ and the methionine synthase gene $M S Y 1$ were also obtained. Both the $c b l 1$ and msyl deletion mutants were methionine auxotrophic and significantly reduced in virulence on corn silks and wheat heads. We also identified genes disrupted by the transforming DNA in three other REMI mutants exhibiting reduced virulence. In mutants M68, the transforming vectors were inserted in the NADH: ubiquinone oxidoreductase. The putative b-ZIP transcription factor gene and the transducin beta-subunit-like gene disrupted in mutants M7 and M75, respectively, had no known homologs in filamentous fungi and were likely to be novel fungal virulence factors.

Additional keywords: auxotrophic mutant, cystathionine, Gibberella zeae, methionine.
Fusarium head blight (FHB) or scab is a disease of wheat and barley that can reduce crop yield and grain quality (28). In North America, the primary pathogen causing this disease is Fusarium graminearum Schwabe (teleomorph Gibberella zeae), lineage 7 $(31,32)$. F . graminearum also causes stalk and ear rots of maize and infects other small grains $(28,34,44)$. Infested cereals may be contaminated with trichothecenes and estrogenic mycotoxins that are harmful to humans and animals and make the grain unsuitable for food or feed $(9,28,33)$. Trichothecene toxins, such as deoxynivalenol (DON), are potent inhibitors of eukaryotic protein biosynthesis.

$F$. graminearum overwinters in infected plant debris as saprophytic mycelia and produces spores in the spring when weather conditions favor fungal development. Ascospores are believed to be the primary inoculum for the disease and are forcibly discharged from mature perithecia and dispersed by wind $(12,41)$. Although it is naturally a haploid homothallic ascomycete, heterothallic $F$. graminearum strains have been generated by deleting the MAT1-1 or MAT1-2 mating-type locus (22). F. graminearum is amenable to classical and molecular genetic manipulations $(4,36)$. Thousands of expressed sequence tags (ESTs) (42) and the genome sequence of $F$. graminearum are now available (provided online by the Broad Institute, Massachusetts Institute of Technology [MIT]). However, most of the molecular studies in this fungus have focused on trichothecene biosynthesis and population structure $(5,7,27,32,45,48)$. There are only limited studies on identification and characterization of fungal virulence factors in F. graminearum (7).

Corresponding author: J.-R. Xu; E-mail address: jinrong @ purdue.edu

DOI: 10.1094/PHYTO-95-0744

(c) 2005 The American Phytopathological Society
The first virulence factor characterized in F. graminearum was the TRI5 trichodiene synthase gene that catalyzes the first committed step in the trichothecene biosynthetic pathway (36). The tri5 deletion mutants are normal in growth and development but reduced in virulence on seedlings of Wheaten wheat, common winter rye, and maize $(8,9,16)$. Further studies with tri5 mutants indicate that DON production is not necessary for initial infection but is important for allowing the fungus to spread within colonized spikes (1). In addition to TRI5, two mitogen-activated protein kinase genes, MGV1 and GPMK1, have been shown to be essential for pathogenicity of $F$. graminearum $(18,19,43)$. The $m g v 1$ deletion mutants are substantially reduced in virulence and DON production, and rarely spread beyond the inoculated floret (18). The mgvl mutant is female sterile and self-incompatible in heterokaryon formation. The gpmkl mutants have no defect in vegetative growth and conidiation, but they are defective in colonizing flowering wheat heads and in spreading from inoculated florets to neighboring spikelets $(19,43)$. Disruption of the homo$\log$ of Cochliobolus heterostrophus CPS1, which represents a novel virulence factor in fungal pathogens, also causes reduced virulence in $F$. graminearum (23).

Restriction enzyme-mediated integration (REMI) mutagenesis has been used in several plant-pathogenic fungi, including Ustilago maydis, C. heterostrophus, and Magnaporthe grisea (2,3, 24,40). REMI remains one of the most efficient approaches for identifying pathogenicity genes in several fungi $(15,21)$. In this study, we generated and screened 6,500 REMI transformants of $F$. graminearum. Eleven pathogenicity mutants defective in colonizing corn silks and flowering wheat heads were isolated. Genetic analyses indicated that the defects in plant infection were tagged by the transforming vector in six of these mutants. In mutant M8, the transforming vector was inserted in the cystathionine beta-lyase gene $(C B L 1)$. We further generated mutants 
deleted for $C B L 1$ and the methionine synthase gene $M S Y 1$, and demonstrated that methionine synthesis is an important virulence factor in $F$. graminearum. In addition, we have identified genes disrupted by the transforming DNA in three other REMI mutants. Two of them appeared to be novel fungal virulence factors in F. graminearum.

\section{MATERIALS AND METHODS}

Strains and culture condition. $F$. graminearum wild-type strain PH-1 (NRRL 31084) and all the mutants (Table 1) generated in this study were cultured at $25^{\circ} \mathrm{C}$ on V8 agar or liquid carboxymethylcellulose (CMC) medium for conidiation (18). A nit1 mutant of PH-1 (11622) was obtained from R. Bowden at Kansas State University. For genomic DNA and RNA extraction, mycelia were cultivated in complete medium (CM) (17) for 3 days at $25^{\circ} \mathrm{C}$. Standard molecular biology procedures were followed for Southern blot analyses (38). CM supplemented with hygromycin B (Calbiochem, La Jolla, CA) at $150 \mu \mathrm{g} / \mathrm{ml}$ or neomycin (Amresco, Solon, OH) at $200 \mu \mathrm{g} / \mathrm{ml}$ was used for selecting transformants. All the REMI mutants were preserved by resuspending conidia and mycelia mixtures scraped off V8 agar culture in $15 \%$ glycerol and stored at $-80^{\circ} \mathrm{C}$. For assaying auxotrophic phenotypes, minimal medium (MM) (17) was supplemented with methionine at $0.5 \mathrm{mg} / \mathrm{ml}$ or 0.5 homocysteine at $\mathrm{mg} / \mathrm{ml}$. Genetic crosses and random ascospore progeny isolation were performed as described previously (18).

Fungal transformation and REMI mutants. Protoplast preparation and fungal transformation were performed as described previously (18). For REMI mutagenesis, plasmids pCB1003 (6) and pKY37 were digested with BamHI and EcoRI, respectively, and directly used for transforming $\mathrm{PH}-1$ protoplasts. To generate pKY37, the hygromycin phosphotransferase $(h p h)$ gene was amplified from pCB1003 with primers 5'-AAGAATTCGGCGCGCCGTCGACGCTGGAGCTAGTGGAGGTCA-3' and 5'-TTGGATCCGGCCGGCCGTCGACCGGTCGGCATCTACTCTATTS$3^{\prime}$. The $\beta$-lactamase gene ( $\left.a m p\right)$ and replication origin (ori) were amplified from pUC19 with primers 5'-AGAATTCGGCGCGCCATCGATGCCTCGTGATACGCCTATTT-3' and 5'-CCGGAATTCATCGATACGAAAACTCACGTTAAGGG-3', and 5'-CCGGAATTCGGATCCGATATCCAGACCCCGTAGAAAAGATC-3' and 5'-TTGGATCCGGCCGGCCGATATCGGGGATAACGCAGGAAAGAA-3', respectively. The amplified $h p h$, amp, and ori fragments were digested with AscI-FseI, AscI-EcoRI, and EcoRIFseI, respectively, and then ligated to construct plasmid pKY36. A polylinker generated by annealing oligoes $5^{\prime}$-AATTCTGCAGAGCTCGAGGTACCTCTAGAAGCTTGTCGACG-3' and 5'GATCCGTCGACAAGCTTCTAGAGGTACCTCGAGCTCTGCAG-3' was inserted between the EcoRI and BamHI sites of pYK36. In the resulting plasmid pKY37, PstI, SacI, XhoI, KpnI, XbaI, HindIII, EcoRI, and BamHI had single digestion sites and were suitable for plasmid rescue. For complementation assays, the wild-type $C B L 1$ and $M S Y 1$ genes were reintroduced into the corresponding mutants by cotransformation with pSM334 (provided by S. Kang at Pennsylvania State University), a vector containing the neomycin phosphotransferase as described previously (18). Neomycin-resistant transformants were screened by polymerase chain reaction (PCR) with primers K1R and $13 \mathrm{~F}$ (18).

Corn silk infection assay. Fresh corn silks were collected from young corn ears of cv. LH 132 and sliced into 5-cm fragments. Exposed portions of corn silks were discarded and only the portions originating from inside husks were used for infection assays. Three to four pieces of corn silk fragments were aligned with each other evenly as a bundle over a Whatman No. 1 filter paper soaked with sterile distilled water. The lower ends of corn silks were covered with a 4-mm ${ }^{2}$ block of $F$. graminearum V8 agar culture. Infection of corn silks was scored by the extent of discoloration after incubation at $25^{\circ} \mathrm{C}$ in a moisture chamber for 4 to 5 days.
Infection assays on flowering wheat inflorescence. Conidia were collected from 7- to 10-day-old cultures on mung bean agar and resuspended in sterile distilled water to a concentration of $10^{6}$ conidia per $\mathrm{ml}$ (13). Approximately 6-week-old plants of wheat cv. Norm were chosen for inoculation at flowering. The fifth full-sized spikelet from the base of the inflorescence was injected with $10 \mu \mathrm{l}$ of the spore suspension containing $0.01 \%$ ( $\mathrm{vol} / \mathrm{vol}$ ) Tween 20; control plants were inoculated with $10 \mu \mathrm{l}$ of $0.01 \%$ Tween alone. Inoculated plants were placed in a humidity chamber for 3 days and then transferred to ambient growth conditions in a greenhouse. Symptomatic spikelets in each head were counted 14 days after inoculation. Student's $t$ tests (two-tailed, two sample unequal variance) were used to compare the mean number of symptomatic spikelets for plants inoculated with $\mathrm{PH}-1$ or mutant strains. The number of inoculated wheat heads per treatment was 9 or 10 for each test, and all tests were repeated at least four times.

Generating the $\boldsymbol{c b l l}$ deletion mutant. To construct the $C B L 1$ gene replacement vector, a $0.6-\mathrm{kb}$ upstream flanking sequence of $C B L 1$ was amplified with primers cb1 (5'-CGCGGATCCTCGATGGGCCATGATAGTGT-3') and cb2 (5'-AAGGTTGGCGCGCCTGGATGCAGGATGTAGTAG-3') and digested with AscI. A 0.7 -kb downstream fragment was amplified with primers cb3 (5'AAGGTTGGCCGGCCTTCAAGCTGGTGCTGTCACT-3') and cb4 (5'-CGCTCTAGAGAGTTTGTGTGGAAGCGTTG-3') and digested with $F s e I$. These flanking sequences of $C B L 1$ were then ligated with a 1.4-kb AscI-FseI hph released from pCX62 (49). Using the ligation mixture as the template, a $2.7-\mathrm{kb}$ fragment containing the gene replacement construct was amplified with primers cb1 and cb4 and cloned between BamHI-XbaI sites on pBCSK as pKY41. pKY41 was digested with BamHI and transformed into $\mathrm{PH}-1$ protoplasts. The resulting transformants were screened by PCR with primers cb5 (5'-ATTCAACCCTCTGGATGTGG-3') and cb6 (5'-TCTGACAAGGCATGCTGATC- $3^{\prime}$ ). All putative cbll gene replacement mutants were further confirmed by Southern blot analyses. For complementation of the cbll mutant, a 4.7-kb XhoI fragment containing the full-length CBL1 gene was amplified with primers cb7 (5'-CTCGAGCGAATGAATCGGAAC-3') and cb8 (5'-CTCGAGCTGCCACCCAGAGTC-3') and cloned into the pGEM-T easy vector (Promega, Madison, WI) as pKY49. Primers cb9 (5'-CGTTCCAAAGCTACTC-3') and cb10 (5'-CGCACTAGGGTGTACA-3') are 408- and 118-bp upstream from the $C B L 1$ open reading frame (ORF), respectively.

Generating the msyl deletion mutant. To generate the MSY1 gene replacement construct, a $0.9-\mathrm{kb}$ upstream fragment was amplified with primers $\mathrm{m} 1$ (5'-ATCGGATCCTTTTCTGATGCCCTGAGAGC-3') and m2 (5'-AAGGTTGGCGCGCCCAAG-

TABLE 1. Pathogenicity phenotypes of restriction enzyme-mediated integration mutants

\begin{tabular}{lcc}
\hline Strain & $\begin{array}{c}\text { Disease index } \\
(\text { mean } \pm \text { SD) }\end{array}$ & $t$ test significance \\
\hline PH-1 & $8.4 \pm 1.4$ & Wild-type control \\
CX8 & 0 & $<0.001$ \\
M28 & 0 & $<0.001$ \\
M30 & 0 & $<0.001$ \\
M75 & 0 & $<0.001$ \\
25C3 & $0.5 \pm 0.5$ & $<0.001$ \\
CX3 & $0.1 \pm 0.1$ & $<0.001$ \\
222 & $0.8 \pm 0.6$ & $<0.001$ \\
2G8 & $0.2 \pm 0.4$ & $<0.001$ \\
M68 & $1.0 \pm 0.3$ & 0.01 \\
M7 & $4.7 \pm 0.7$ & 0.006 \\
M8 & $3.7 \pm 1.0$ & 0.002 \\
\hline
\end{tabular}

${ }^{a}$ Disease index is represented by the mean and standard deviation of numbers of symptomatic spikelets at 14 days after inoculation in four independent infection assays. 
GTTGGTTAGTCGTTG-3') and digested with AscI. A 0.7-kb downstream fragment amplified with primers m3 (5'-AAGGTTGGCCGGCCTGTGTGTAGAGGTTGATGGG-3') and $\mathrm{m} 4$ (5'ATCAAGCTTTTGTACCTTCGGCTTCAGCT-3') was digested with FseI. After ligation of these PCR products with the AscI-FseI $h p h$ fragment, a 3.0-kb MSY1 gene replacement construct was amplified with primers $\mathrm{m} 1$ and $\mathrm{m} 4$ and cloned into pGEM-T easy as pKY43. After being linearized with EcoRI, pKY43 was transformed into protoplasts of PH-1. Hygromycin-resistant transformants were isolated and examined for their growth on MM with or without methionine. Putative methionine auxotrophic msyl mutants were further confirmed by Southern hybridizations with probe 1 amplified with primers $\mathrm{m} 1 / \mathrm{m} 2$ and probe 2 amplified with primers $\mathrm{m} 5 / \mathrm{m} 6$. For complementation of the msyl mutant, a $4.3-\mathrm{kb}$ fragment containing the $M S Y 1$ gene was amplified with primers $\mathrm{m} 7$ (5'-AGGCATTGCAATGCCACAAC-3') and m8 (5'-TTCAGACAGTGACGCATCAC- $3^{\prime}$ ) and cloned into pGEM-T easy as pKY52.

GenBank accession numbers are as follows: EAA68828 for CBL1 (FG01932.1), EAA75179 for MSY1 (FG10825.1), EAA68510 for ZIF1 (FG01555.1), EAA69636 for NOS1 (FG00376.1), and EAA67363 for TBL1 (FG00332.1).

\section{RESULTS}

Generating REMI transformants. We isolated 6,500 hygromycin-resistant transformants by transforming PH-1 with either BamHI-digested pCB1003 or EcoRI-digested pKY37. To determine the integration events in these transformants, 22 randomly selected pKY37 transformants were analyzed by Southern blot hybridization. When blots of DNA samples digested with HindIII were probed with the $h p h$ fragment, 13 transformants showed a single band larger than $3.3 \mathrm{~kb}$ (Fig. 1) and likely contained one copy of pKY37 integrated at a single site. Seven of these transformants had a 3.3-kb band and one additional band (Fig. 1), indicating that more than one copy of pKY37 was tandem integrated at a single site. The single $3.3-\mathrm{kb}$ band observed in transformant 7 (Fig. 1) was likely a doublet band. Two $(<10 \%)$ of them, transformants 13 and 19, had two or more bands in addition to the $3.3-\mathrm{kb}$ band (Fig. 1). When blots of StuI-digested DNAs were probed with the $h p h$ gene, transformants 13 and 19 had more than one band (data not shown), suggesting that pKY37 was inserted at two different loci in these two transformants. Similar Southern blot analyses with 20 randomly selected pCB1003 transformants indicated that 18 of them $(90 \%)$ had the transforming vector integrated at a single genomic site (data not shown).
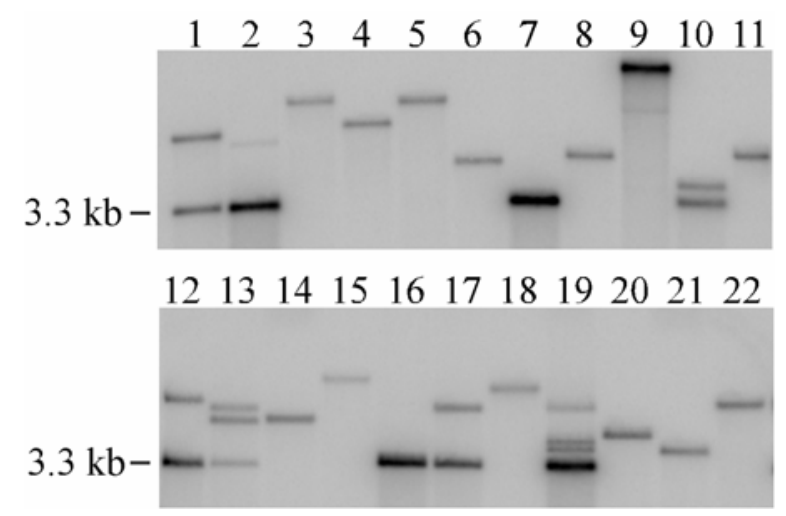

Fig. 1. Southern blot analyses of restriction enzyme-mediated integration (REMI) transformants. Blots of HindIII-digested DNAs from 22 randomly selected REMI transformants ( 1 to 22 ) were hybridized with the $h p h$ gene. Nine transformants had the $3.3-\mathrm{kb}$ band resulting from the tandem integration of more than one copy of the transforming vector pKY37, which had a single HindIII site.
Screening for mutants defective in plant infection. To screen for mutants defective in plant infection, we devised a corn silk infection assay as described previously. $F$. graminearum is a causal agent of the corn ear rot disease and can infect corn kernels through silks $(16,37)$. The wild-type strain PH-1 colonized fresh corn silks and caused discoloration (Fig. 2A). The corn silk infection was used as the primary screen to assay the virulence of all 6,500 REMI transformants. REMI mutants identified in the primary screen were tested in three additional corn silk infection assays. Only 20 transformants with reduced virulence in repeated corn silk infection assays were used to inoculate flowering wheat heads. Table 1 lists 11 REMI mutants that were significantly reduced in virulence on both corn silks and flowering wheat heads (Table 1). REMI mutants CX8 (Fig. 2B), M28, M30, and M75 usually did not cause any symptoms in inoculated florets (Table 1). Mutants 25C3 (Fig. 2B), CX3, 222, 2G8, and M68 colonized the inoculated florets but usually failed to spread to other florets on the same spikelets (Table 1). In contrast, mutants M7 (Fig. 2B) and M8 were still pathogenic but the number of infected kernels was significantly reduced in inoculated wheat heads, indicating that these REMI mutants were less virulent and might be defective in spreading through infected wheat heads (Table 1).

These REMI mutants were crossed with the nitl mutant strain 11622. Outcross perithecia were identified by the ability of ascospore progeny to grow on MM with hygromycin and $1 \%$ potassium chlorate. Random mono-ascospore cultures were isolated from outcross perithecia and assayed for their resistance to hygro-

$\mathbf{A}$

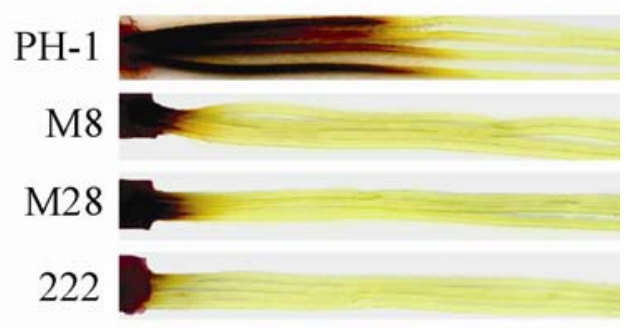

B
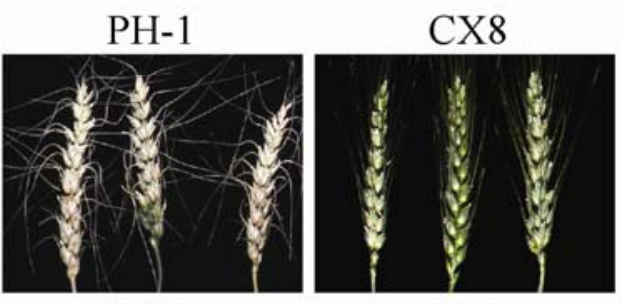

$25 \mathrm{C} 3$
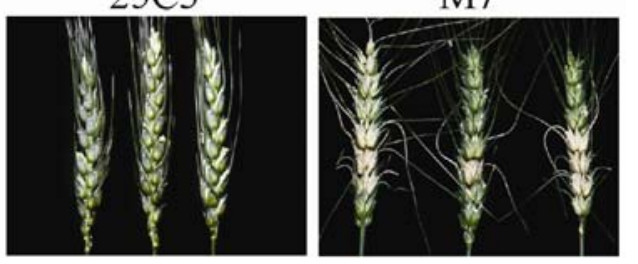

Fig. 2. Restriction enzyme-mediated integration (REMI) mutants with reduced virulence. A, Corn silk infection assay. A small block of V8 agar cultures of wild-type PH-1 and REMI mutants M8, M28, and 222 were placed on freshly cut ends of corn silks. PH-1 colonized and caused discoloration in infected corn silks. Discoloration was significantly reduced in mutants M8, M28, and 222. Photos were taken 4 days after inoculation. B, Infection assay on wheat heads. Scab disease development on wheat heads inoculated in the fifth spikelet from the bottom with conidia from the wild-type strain (PH-1) and REMI mutants (25C3, CX8, and M7). Photos were taken 2 weeks postinoculation. Mutants CX8 and 25C3 were reduced in their ability to cause necrosis on wheat kernels beyond the point of inoculation. 
mycin and virulence on wheat plants and corn silks (data not shown). Cosegregation analysis indicated that the phenotypes of REMI mutants CX3, 222, M7, M8, M68, and M75 were tagged by the transforming hygromycin-resistant vector. In crosses between 11622 and CX8, 2G8, M28 or M30, we had isolated progeny that were sensitive to hygromycin but displayed mutant phenotypes, indicating that defects observed in these mutants were not caused by transforming DNA. Cosegregation analysis was not performed with mutant $25 \mathrm{C} 3$ because it contained multiple integrations of the transforming vector (data not shown).

Mutant M8 was disrupted in the cystathionine $\beta$-lyase gene. Mutant M8 grew at a normal rate on V8 agar plates but was significantly reduced in virulence (Table 1 ). To identify the gene disrupted in M8, genomic DNA was digested with XhoI and transformed into Escherichia coli after self-ligation. Five identical clones containing a 2.2-kb EcoRI-XhoI fragment of F. graminearum genomic DNA were recovered, and a representative was designated pKY40 (Fig. 3A). Sequence analysis of the fragment recovered in pKY40 indicated that the transforming vector pKY37 was inserted at the EcoRI site located 110-bp upstream from the start codon of the $C B L 1$ (for cystathionine beta-lyase 1) gene (Fig. 3A). CBL1 is highly homologous to the Neurospora crassa MET-2 and Aspergillus nidulans met $G$ genes. We searched the available $F$. graminearum EST sequences but could not identify the CBL1 cDNA sequence. However, using first-strand cDNA synthesized from RNA of PH-1 mycelia grown on CM, a 290-bp fragment upstream from the CBL1 ORF ( -408 to -118 ) was amplified with primers $\operatorname{cb} 9$ and cb10 (data not shown). Therefore, the transcription of $C B L 1$ must be initiated upstream from the integration site of pKY37 in mutant M8. The insertion of pKY37 was in the $5^{\prime}$-untranslated region and likely abolished the expression of the $C B L 1$ gene.

To confirm that the phenotype of M8 was caused by the disruption of $C B L 1$ expression, we generated a $C B L 1$ gene replacement construct pKY41 (Fig. 3A) and transformed it into PH-1. Two cbll gene replacement mutants, $\mathrm{KC} 1$ and $\mathrm{KC} 2$, were identified and confirmed by Southern blot analyses (Fig. 3B). The wild-type strain PH-1 had a 1.6-kb EcoRI and a 1.8-kb SalI band when hybridized with probe 1 and probe 2 , respectively. The cbll mutants $\mathrm{KC} 1$ and $\mathrm{KC} 2$ had no signal when hybridized with probe 1 (Fig. 3B) and contained a 5.1-kb Sall band when hybridized with probe 2. Similar to REMI mutant M8, the cbll deletion mutant was significantly reduced in plant infection (Fig. 4A). The colony formed by the cbll mutant was morphologically identical to that of M8 and had much less aerial hyphae than PH-1 (Fig. 4B). Similar to the A. nidulans mutants that are blocked in conversion of cystathionine to homocysteine (39), mutant M8 and cbll mutants were methionine auxotrophic (Fig. 4C). Supplementation of homocysteine or methionine rescued the growth defect of M8 and the cbll deletion mutants on MM. When a wild-type $C B L 1$ gene was cotransformed into the cbll mutant $\mathrm{KC1}$, the resulting transformants $\mathrm{KCC} 1$ and $\mathrm{KCC} 2$ harboring $\mathrm{pKY} 49$ were prototrophic and normal in virulence on corn silks and flowering wheat heads (data not shown).

Deletion of the methionine synthase gene also reduced virulence in $\boldsymbol{F}$. graminearum. Since the cystathionine $\beta$-lyase catalyzes the conversion of cystathionine to homocysteine, which is a precursor for methionine synthesis, we also generated a gene replacement construct pKY43 (Fig. 5A) for deleting the methionine synthase gene $M S Y 1$ identified in the $F$. graminearum genome sequence. After transformation of PH-1 with pKY43, 25 hygromycin-resistant transformants were isolated and screened for their inability to grow on MM without methionine. Two methionine auxotrophic transformants, KM1 and KM2, were identified and confirmed by Southern blot analyses to be deleted of the MSY1 gene (Fig. 5B). Similar to the cbll mutant, the msyl mutants were defective in wheat head infection (Fig. 4A) and methionine auxotrophic (Fig. 4C), indicating that defects in methionine synthesis significantly reduced the virulence in $F$. graminearum. The cbll and msyl mutants had no defects in conidiation in the liquid CMC medium (data not shown).

Although both msyl and cbll mutants displayed methionine auxotrophic phenotypes, in contrast to the cbll mutant, the msyl deletion mutant failed to grow on MM supplemented with homocysteine (Fig. 4C). The colony morphology of the msyl mutant was also distinct from that of the cbll mutant. On V8 agar or MM plus methionine plates, the msyl mutants produced much less aerial hyphae than the cbll mutants (Fig. 4B and C). When the wild-type MSY1 gene was amplified and re-introduced into the msyl mutant KM2 by cotransformation, the resulting transformant KMC1 harboring pKY52 was prototrophic and normal in plant infections. Transformant KMC1 was also normal in colony morphology and aerial hyphae growth (data not shown). The difference between the msyl and cbll mutants in aerial hypha production, even in the presence of methionine, indicated that robust aerial hyphal growth requires the methionine synthase gene.

Genes disrupted in other REMI mutants. To identify genes disrupted by the transforming vector, genomic DNAs of M7, M68, and M75 were digested by PstI, HindIII, and KpnI, respectively, and transformed into $E$. coli after self-ligation. Sequence analyses of the genomic fragments recovered by plasmid rescue indicated that the transforming vector pKY37 was integrated in mutants M7, M68, and M75 at the recognition site of EcoRI, which was the restriction enzyme used in transformation to generate these mutants.

The insertion site of pKY37 in mutant M7 was located at the 3' end (amino acid residue 487) of a putative b-ZIP transcription factor gene FG01555.1 (named ZIF1 for bZIP transcription factor $\underline{1}$ ).

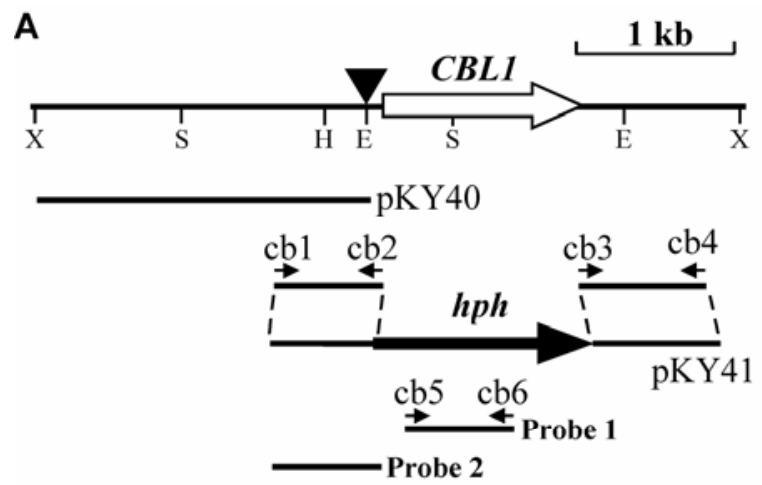

B

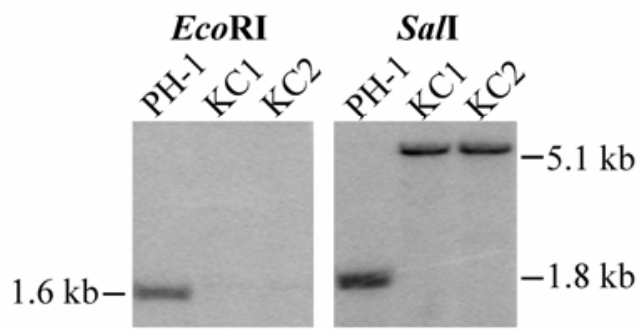

Fig. 3. The $C B L 1$ locus of mutant M8 and gene replacement of $C B L 1$. A, The filled triangle indicated the EcoRI site where pKY37 was inserted upstream of $C B L 1$ in M8. The XbaI-EcoRI fragment was recovered from M8 in the plasmid rescue clone pKY40. The $C B L 1$ gene replacement vector pKY41 was generated by ligating a $0.6-\mathrm{kb}$ upstream and a $0.7-\mathrm{kb}$ downstream flanking sequence of $C B L 1$ with the $h p h$ gene. The empty and filled arrows indicate the direction of the $C B L 1$ and $h p h$ genes, respectively. The positions and directions of primers $\mathrm{cb} 1, \mathrm{cb} 2, \mathrm{cb} 3, \mathrm{cb} 4, \mathrm{cb} 5$, and $\mathrm{cb} 6$ are indicated with small arrows. Probe 1 and probe 2 indicate polymerase chain reaction products amplified with primers $\mathrm{cb} 1 / \mathrm{cb} 2$ and $\mathrm{cb} 5 / \mathrm{cb} 6$, respectively. Restriction enzymes BamHI (B), EcoRI (E), HindIII (H), PstI (P), SacI (S), XhoI (X), and XbaI $(\mathrm{Xb})$. B, Southern blots of the wild-type strain $\mathrm{PH}-1$ and the cbll deletion mutants $\mathrm{KC} 1$ and $\mathrm{KC} 2$. DNA samples were digested with EcoRI and hybridized with probe 1 (left) or digested with SalI and hybridized with probe 2 (right). 
The protein encoded by ZIF1 contained a basic region leucine zipper domain. In mutant M68, the insertion site was 103-bp upstream from the ORF of the predicted gene FG00376.1 (named NOS1) that is homologous to the $49-\mathrm{kDa}$ subunit of NADH: ubiquinone oxidoreductase (complex I) from Neurospora crassa (30). In mutant M75, pKY37 was integrated at amino acid residue

A

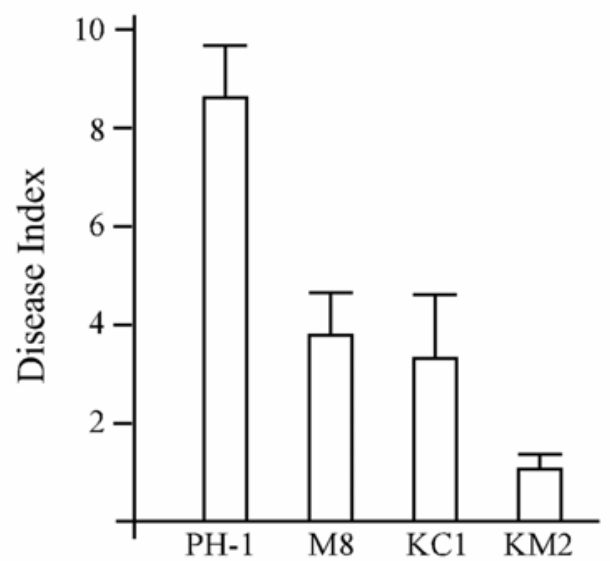

B

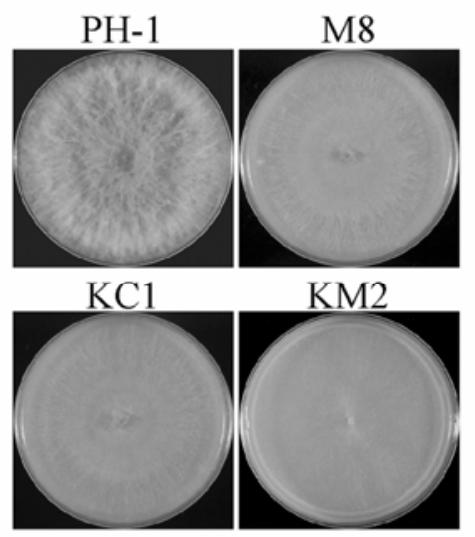

C

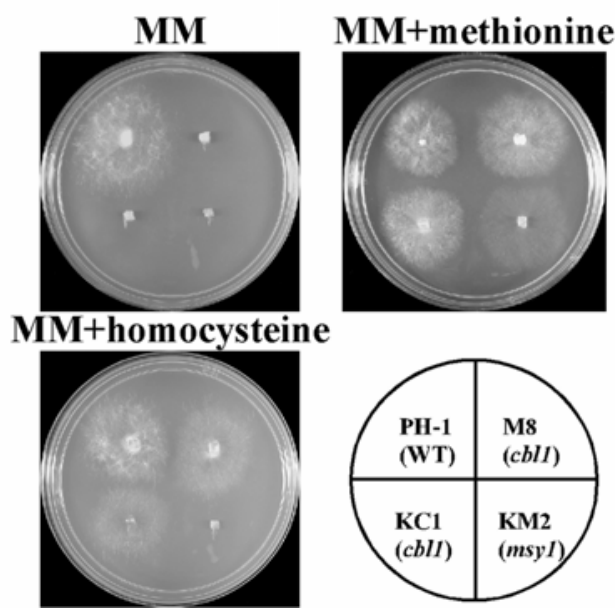

Fig. 4. The cbll and msyl mutants were defective in plant infection and methionine auxotrophic. A, Flowering wheat heads were inoculated with conidia from $\mathrm{PH}-1$, restriction enzyme-mediated integration (REMI) mutant M8, and the cbll (KC1) or msyl (KM2) deletion mutants. Disease index is represented by the mean and standard deviation of numbers of symptomatic spikelets in four independent infection assays. Virulence of the cbll and msyl mutants, similar to that of mutant M8, was significantly reduced. $\mathbf{B}, \mathrm{V} 8$ agar cultures of the wild-type strain PH-1, REMI mutant M8, and the cbll and msyl deletion mutants $\mathrm{KC} 1$ and $\mathrm{KM} 2$, respectively. $\mathbf{C}$, The same set of strains grown on minimal medium (MM) and MM supplemented with methionine (MM+M) or homocysteine $(\mathrm{MM}+\mathrm{H})$. Photos were taken after 5 days of incubation at $25^{\circ} \mathrm{C}$.
6 of the predicted ORF FG00332.1 (named TBL1 for transducin beta-subunit-like gene 1), which encodes a 662-amino acid protein homologous to the beta-subunits of transducins $(11,25)$. The TBL1 gene product has seven WD repeats (14) at the $\mathrm{C}$ terminus and an $\mathrm{N}$-terminal LisH domain that is known to be involved in protein-protein interactions (10).

\section{DISCUSSION}

REMI has been used in several filamentous fungi for insertional mutagenesis $(15,21)$. Although the random nature of plasmid integration is a matter of debate, REMI has been used effectively in recovering novel virulence factors in many plant-pathogenic fungi $(3,24,40)$. In this study, we isolated 11 pathogenicity mutants after screening 6,500 F. graminearum REMI transformants. Approximately $90 \%$ of the REMI transformants were estimated to have the transforming vector integrated at a single locus. In M. grisea, it has been shown that removal of polyethylene glycol (PEG) during regeneration favors single-plasmid insertion events at endonuclease-cleaved sites (2). In our protocol, PEG added to the transformation mixtures was removed by centrifugation before the protoplasts were plated out in regeneration media for selecting hygromycin-resistant transformants.

Since wheat head infection assays are time-consuming and labor-intensive, we devised a corn silk infection assay. Our data suggested that the corn silk infection assay is useful as the primary screen to rapidly identify mutants that are defective in wheat

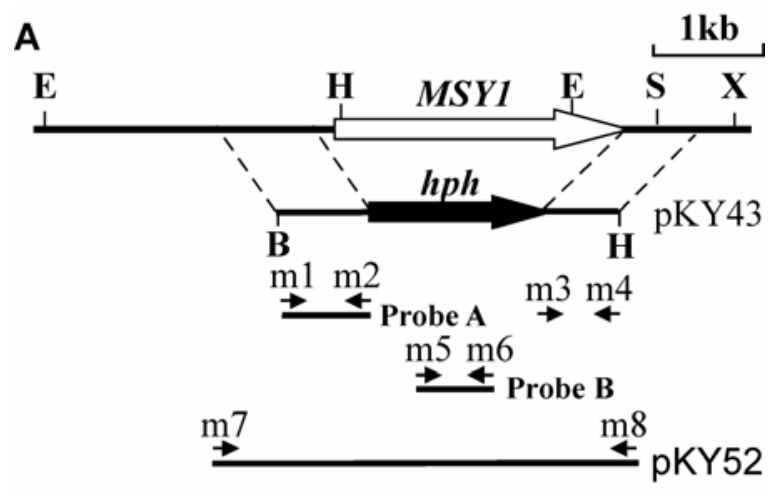

B

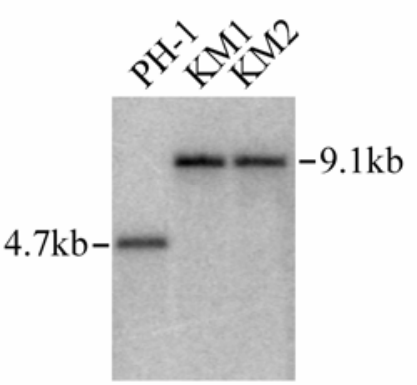

Probe A

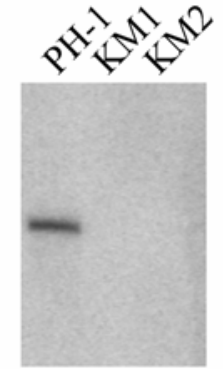

Probe B
Fig. 5. Gene replacement of the methionine synthase MSY1. A, The gene replacement construct pKY43 was generated by ligating a $0.9-\mathrm{kb}$ upstream and a $0.7-\mathrm{kb}$ downstream flanking sequence of $M S Y 1$ with the $h p h$ gene. The empty and filled arrows indicate the direction of the MSY1 and hph genes, respectively. The positions and directions of primers $\mathrm{m} 1, \mathrm{~m} 2, \mathrm{~m} 3, \mathrm{~m} 4, \mathrm{~m} 5$, $\mathrm{m} 6, \mathrm{~m} 7$, and $\mathrm{m} 8$ are indicated with small arrows. Probe A and probe B are polymerase chain reaction products amplified with primers $\mathrm{ms} 1 / \mathrm{ms} 2$ and ms5/ms6, respectively. BamHI (B), EcoRI (E), HindIII (H), SacI (S), XhoI (X). B, Southern blots of EcoRI-digested genomic DNAs of PH-1, the msyl deletion mutants KM1 and KM2 were hybridized with probe A (left) and probe B (right). The msy1 mutants KM1 and KM2 had a 9.1-kb EcoRI band that hybridized with probe A but no signal when hybridized with probe $\mathrm{B}$. The wild-type strain PH-1 had a 3.7-kb EcoRI band when hybridized with both probes. 
head infection. The mgvl and gpmkl mutants $(18,19)$ that are known to be defective in wheat infection were nonpathogenic on corn silks (data not shown). However, not all the REMI transformants that were defective in colonizing corn silks were significantly reduced in infecting wheat heads. In fact, 9 out of 20 REMI mutants with reduced virulence on corn silks were as virulent as the wild-type strain when assayed on flowering wheat heads (data not shown). It is possible that different virulence factors in F. graminearum are involved in infecting corn silks versus wheat heads. However, we attempted to include all putative virulence mutants identified in the primary screens for wheat head infection assays. Some of them were only slightly reduced in virulence on corn silks and possibly not true mutants defective in plant infection. On the other hand, it is also possible that some F. graminearum genes are specific for wheat head infection. Mutants disrupted in this type of gene are likely to be normal in colonizing corn silks and unidentified in the primary screen with corn silk infection assays.

In mutant M8, the transforming vector was inserted 110-bp upstream from the start codon of the $C B L 1$ gene. The transforming vector might have disrupted the transcription of the $C B L 1$ gene resulting in the methionine auxotrophic phenotype of mutant M8. Mutants deleted of the CBL1 or the methionine synthase gene $M S Y 1$ were, similar to M8, significantly reduced in virulence on corn silks and flowering wheat heads, indicating that methionine synthesis is critical for plant infection in $F$. graminearum. In $M$. grisea, the methionine auxotrophic mutant 130 (met 1 ) was also reduced in virulence on rice seedlings (2). However, it is not clear which gene was disrupted in this mutant. In Cryptococcus neoformans, mutants deleted of the methionine synthase gene MET6 are nonpathogenic (35). Auxotrophic mutants of other fungal pathogens defective in other primary metabolism, such as histidine synthesis in $M$. grisea (40) and arginine synthesis in F. oxysporum (29), have also been reported to be reduced in virulence.

Although both the cbll and the msyl mutants were methionine auxotrophic, we noticed that the msyl mutant was more severely reduced than the cbll mutant in aerial hyphal growth and virulence (Fig. 4). In N. crassa and A. nidulans, homocysteine can be synthesized from homoserine by homoserine acetyltransferase and homocysteine synthase (20). Homologs of the homoserine acetyltransferase and homocysteine synthase genes found in the F. graminearum genome (FG05658.1 and FG01417.1) may be functional and responsible for homocysteine synthesis in the cbll deletion mutant and REMI mutant M8. The msyl mutant displayed a growth defect on MM supplemented with methionine (Fig. 4C). A similar phenomenon has been reported in $C$. neoformans $(35,47)$. The met 3 mutants of $C$. neoformans blocked in the sulfate-assimilation arm of the methionine biosynthetic pathway are reduced in virulence and have a growth defect even in the presence of abundant exogenous methionine (47). The met6 mutant also grew poorly in media supplemented with methionine (35). It is possible that deletion of the methionine synthase gene leads to the accumulation of homocysteine, a toxic intermediate that interferes with ergosterol biosynthesis in F. graminearum $(26,35)$.

In addition to M8, we have identified genes disrupted in mutants M7, M68, and M75. The growth rates of these REMI mutants were similar to that of the wild-type strain (data not shown). The putative b-ZIP transcription factor gene ZIFI disrupted in mutant M7 had no significant homology in yeasts and appeared to be unique to filamentous fungi. The TBL1 gene disrupted in mutant M75 is highly conserved among filamentous fungi, including $N$. crassa, A. nidulans, and M. grisea. However, all of its homologs are predicted genes that have not been functionally characterized. Different from the G-beta subunits characterized in various fungi, the transducin beta-subunit-like protein has the LisH domain. In A. nidulans, NudF has the LisH domain and is required for nuclear movement (46). However, the
F. graminearum genome has distinct homologs of the G-beta subunit and $N u d F$ genes that are not related with $T B L 1$. The wildtype ZIF1 and TBL1 genes were transformed into mutants $\mathrm{M} 7$ and M75, respectively. Preliminary analyses indicated that re-introduction of the wild-type alleles of ZIF1 and TBL1 complemented the phenotypes of mutants M7 and M75, respectively (K. Seong and J.-R. Xu, unpublished data). Since there are no homologs of ZIF1 and TBL1 that have been characterized in filamentous fungi, the putative b-ZIP transcription factor and transducin betasubunit-like protein genes identified in this study are likely to be novel fungal virulence factors. It will be important to further characterize the ZIFI and TBLI genes and to determine their roles in plant infection processes.

\section{ACKNOWLEDGMENTS}

We thank L. Dunkle and C. Woloshuk for critical reading of this manuscript, and G. Shaner and G. Buechley for assistance with wheat infection assays. This work was supported by a grant from the U.S. Wheat and Barley Scab initiative to H. C. Kistler and a grant to J.-R. Xu from the U.S. Department of Agriculture-National Research Initiative (200301130). This is journal article no. 17664 of the Purdue University Agriculture Experiment Station.

\section{LITERATURE CITED}

1. Bai, G. H., Desjardins, A. E., and Plattner, R. D. 2002. Deoxynivalenolnonproducing Fusarium graminearum causes initial infection, but does not cause disease spread in wheat spikes. Mycopathologia 153:91-98.

2. Balhadere, P. V., Foster, A. J., and Talbot, N. J. 1999. Identification of pathogenicity mutants of the rice blast fungus Magnaporthe grisea by insertional mutagenesis. Mol. Plant-Microbe Interact. 12:129-142.

3. Bolker, M., Bohnert, H. U., Braun, K. H., Gorl, J., and Kahmann, R. 1995. Tagging pathogenicity genes in Ustilago maydis by restriction enzyme-mediated integration (REMI). Mol. Gen. Genet. 248:547-552.

4. Bowden, R. L., and Leslie, J. F. 1999. Sexual recombination in Gibberella zeae. Phytopathology 89:182-188.

5. Brown, D. W., Dyer, R. B., McCormick, S. P., Kendra, D. F., and Plattner, R. D. 2004. Functional demarcation of the Fusarium core trichothecene gene cluster. Fungal Genet. Biol. 41:454-462.

6. Carroll, A. M., Sweigard, J. A., and Valent, B. 1994. Improved vectors for selecting resistance to hygromycin. Fungal Genet. Newsl. 41:22.

7. Cumagun, C. J. R., Bowden, R. L., Jurgenson, J. E., Leslie, J. F., and Miedaner, T. 2004. Genetic mapping of pathogenicity and aggressiveness of Gibberella zeae (Fusarium graminearum) toward wheat. Phytopathology 94:520-526.

8. Desjardins, A. E., Bai, G. H., Plattner, R. D., and Proctor, R. H. 2000. Analysis of aberrant virulence of Gibberella zeae following transformation-mediated complementation of a trichothecene deficient (Tri5) mutant. Microbiology 146:2059-2068.

9. Desjardins, A. E., Proctor, R. H., Bai, G. H., McCormick, S. P., Shaner, G., Buechley, G., and Hohn, T. M. 1996. Reduced virulence of trichothecene-nonproducing mutants of Gibberella zeae in wheat field tests. Mol. Plant-Microbe Interact. 9:775-781.

10. Emes, R. D., and Ponting, C. P. 2001. A new sequence motif linking lissencephaly, Treacher Collins and oral-facial-digital type 1 syndromes, microtubule dynamics and cell migration. Human Mol. Genet. 10:28132820.

11. Fong, H. K. W., Hurley, J. B., Hopkins, R. S., Miakelye, R., Johnson, M. S., Doolittle, R. F., and Simon, M. I. 1986. Repetitive segmental structure of the transducin beta-subunit-homology with the CDC4 gene and identification of related messenger RNAs. Proc. Natl. Acad. Sci. USA 83:2162-2166.

12. Francl, L., Shaner, G., Bergstrom, G., Gilbert, J., Pedersen, W., DillMacky, R., Sweets, L., Corwin, B., Jin, Y., Gallenberg, D., and Wiersma, J. 1999. Daily inoculum levels of Gibberella zeae on wheat spikes. Plant Dis. 83:662-666.

13. Gale, L. R., Chen, L.-F., Hernick, C. A., Takamura, K., and Kistler, H. C. 2002. Population analysis of Fusarium graminearum from wheat fields in eastern China. Phytopathology 92:1315-1322.

14. Garcia-Higuera, I., Fenoglio, J., Li, Y., Lewis, C., Panchenko, M. P., Reiner, O., Smith, T. F., and Neer, E. J. 1996. Folding of proteins with WD-repeats: Comparison of six members of the WD-repeat superfamily to the G protein beta subunit. Biochemistry 35:13985-13994.

15. Gold, S. E., Garcia-Pedrajas, M. D., and Martinez-Espinoza, A. D. 2001. New (and used) approaches to the study of fungal pathogenicity. Annu. Rev. Phytopathol. 39:337-365. 
16. Harris, L. J., Desjardins, A. E., Plattner, R. D., Nicholson, P., Butler, G., Young, J. C., Weston, G., Proctor, R. H., and Hohn, T. M. 1999. Possible role of trichothecene mycotoxins in virulence of Fusarium graminearum on maize. Plant Dis. 83:954-960.

17. Harris, S. D., Morrell, J. L., and Hamer, J. E. 1994. Identification and characterization of Aspergillus nidulans mutants defective in cytokinesis. Genetics 136:517-532.

18. Hou, Z. M., Xue, C. Y., Peng, Y. L., Katan, T., Kistler, H. C., and Xu, J. R. 2002. A mitogen-activated protein kinase gene (MGV1) in Fusarium graminearum is required for female fertility, heterokaryon formation, and plant infection. Mol. Plant-Microbe Interact. 15:1119-1127.

19. Jenczmionka, N. J., Maier, F. J., Losch, A. P., and Schafer, W. 2003. Mating, conidiation and pathogenicity of Fusarium graminearum, the main causal agent of the head-blight disease of wheat, are regulated by the MAP kinase gpmk1. Curr. Genet. 43:87-95.

20. Kacprzak, M. M., Lewandowska, I., Matthews, R. G., and Paszewski, A. 2003. Transcriptional regulation of methionine synthase by homocysteine and choline in Aspergillus nidulans. Biochem. J. 376:517-524.

21. Kahmann, R., and Basse, C. 2001. Fungal gene expression during pathogenesis related development and host plant colonization. Curr. Opin. Microbiol. 4:374-380.

22. Lee, J., Lee, T., Lee, Y. W., Yun, S. H., and Turgeon, B. G. 2003. Shifting fungal reproductive mode by manipulation of mating type genes: Obligatory heterothallism of Gibberella zeae. Mol. Microbiol. 50:145152.

23. Lu, S. W., Kroken, S., Lee, B. N., Robbertse, B., Churchill, A. C. L., Yoder, O. C., and Turgeon, B. G. 2003. A novel class of gene controlling virulence in plant pathogenic ascomycete fungi. Proc. Natl. Acad. Sci. USA 100:5980-5985.

24. Lu, S. W., Lyngholm, L., Yang, G., Bronson, C., Yoder, O. C., and Turgeon, B. G. 1994. Tagged mutations at the TOX1 locus of Cochliobolus heterostrophus by restriction enzyme-mediated integration. Proc. Natl. Acad. Sci. USA 91:12649-12653.

25. Makino, E. R., Handy, J. W., Li, T. S., and Arshavsky, V. Y. 1999. The GTPase activating factor for transducin in rod photoreceptors is the complex between RGS9 and type 5 G protein beta subunit. Proc. Natl. Acad. Sci. USA 96:1947-1952.

26. McCammon, M. T., and Parks, L. W. 1981. Inhibition of sterol transmethylation by S-adenosylhomocysteine analogs. J. Bacteriol. 145:106112.

27. McCormick, S. P., Harris, L. J., Alexander, N. J., Ouellet, T., Saparno, A., Allard, S., and Desjardins, A. E. 2004. Tri1 in Fusarium graminearum encodes a P450 oxygenase. Appl. Environ. Microbiol. 70:2044-2051.

28. McMullen, M., Jones, R., and Gallenberg, D. 1997. Scab of wheat and barley: A re-emerging disease of devastating impact. Plant Dis. 81:13401348.

29. Namiki, F., Matsunaga, M., Okuda, M., Inoue, I., Nishi, K., Fujita, Y., and Tsuge, T. 2001. Mutation of an arginine biosynthesis gene causes reduced pathogenicity in Fusarium oxysporum f. sp. melonis. Mol. Plant-Microbe Interact. 14:580-584.

30. Nehls, U., Hemmer, S., Rohlen, D. A., Vanderpas, J. C., Preis, D., Sackmann, U., and Weiss, H. 1991. cDNA and genomic DNA sequence of the $21.3 \mathrm{KDa}$ subunit of NADH-ubiquinone reductase (complex-I) from Neurospora crassa. Biochem. Biophy. Acta 1088:325-326.

31. O'Donnell, K., Kistler, H. C., Tacke, B. K., and Casper, H. H. 2000. Gene genealogies reveal global phylogeographic structure and reproductive isolation among lineages of Fusarium graminearum, the fungus causing wheat scab. Proc. Natl. Acad. Sci. USA 97:7905-7910.
32. O'Donnell, K., Ward, T. J., Geiser, D. M., Kistler, H. C., and Aoki, T. 2004. Genealogical concordance between the mating type locus and seven other nuclear genes supports formal recognition of nine phylogenetically distinct species within the Fusarium graminearum clade. Fungal Genet. Biol. 41:600-623.

33. Ozbek, E., and Ozbek, A. 2003. Kidney damage by dietary Fusarium graminearum in rats: A microscopic study. J. Int. Med. Res. 31:529-536.

34. Parry, D. W., Jenkinson, P., and McLeod, L. 1995. Fusarium ear blight (scab) in small grain cereals-A review. Plant Pathol. 44:207-238.

35. Pascon, R. C., Ganous, T. M., Kingsburry, J. M., Cox, G. M., and McCusker, J. H. 2004. Cryptococcus neoformans methionine synthase: Expression analysis and requirement for virulence. Microbiology (UK) 150:3013-3023.

36. Proctor, R. H., Hohn, T. M., and McCormick, S. P. 1995. Reduced virulence of Gibberella zeae caused by disruption of a trichothecene toxin biosynthetic gene. Mol. Plant-Microbe Interact. 8:593-601.

37. Reid, L. M., Woldemariam, T., Zhu, X., Stewart, D. W., and Schaafsma, A. W. 2002. Effect of inoculation time and point of entry on disease severity in Fusarium graminearum, Fusarium verticillioides, or Fusarium subglutinans inoculated maize ears. Can. J. Plant Pathol. 24:162-167.

38. Sambrook, J., Fritsch, E. F., and Maniatis, T. 1989. Molecular Cloning: A Laboratory Manual. 2nd ed. Cold Spring Harbor Laboratory, Cold Spring Harbor, NY.

39. Sienko, M., and Paszewski, A. 1999. The met $G$ gene of Aspergillus nidulans encoding cystathionine beta-lyase: Cloning and analysis. Curr. Genet. 35:638-646.

40. Sweigard, J. A., Carroll, A. M., Farrall, L., Chumley, F. G., and Valent, B. 1998. Magnaporthe grisea pathogenicity genes obtained through insertional mutagenesis. Mol. Plant-Microbe Interact. 11:404-412.

41. Trail, F., Xu, H. X., Loranger, R., and Gadoury, D. 2002. Physiological and environmental aspects of ascospore discharge in Gibberella zeae (anamorph Fusarium graminearum). Mycologia 94:181-189.

42. Trail, F., Xu, J. R., San Miguel, P., Halgren, R. G., and Kistler, H. C. 2003. Analysis of expressed sequence tags from Gibberella zeae (anamorph Fusarium graminearum). Fungal Genet. Biol. 38:187-197.

43. Urban, M., Mott, E., Farley, T., and Hammond-Kosack, K. 2003. The Fusarium graminearum MAP1 gene is essential for pathogenicity and development of perithecia. Mol. Plant Pathol. 4:347-359.

44. White, E. M., McGarel, A. S. L., and Ruddle, O. 2003. The influence of variety, year, disease control and plant growth regulator application on crop damage, yield and quality of winter oats (Avena sativa). J. Agric. Sci. 140:31-42.

45. Wuchiyama, J., Kimura, M., and Yamaguchi, I. 2000. A trichothecene efflux pump encoded by Tri102 in the biosynthetic gene cluster of Fusarium graminearum. J. Antibiot. 53:196-200.

46. Xiang, X., Osmani, A. H., Osmani, S. A., Xin, M., and Morris, N. R. 1995. NudF, a nuclear migration gene in Aspergillus nidulans, is similar to the human $L I S-1$ gene required for neuronal migration. Mol. Biol. Cell 6:297-310

47. Yang, Z. H., Pascon, R. C., Alspaugh, J. A., Cox, G. M., and McCusker, J. H. 2002. Molecular and genetic analysis of the Cryptococcus neoformans MET3 gene and a met3 mutant. Microbiology 148:2617-2625.

48. Zeller, K. A., Bowden, R. L., and Leslie, J. F. 2004. Population differentiation and recombination in wheat scab populations of Gibberella zeae from the United States. Mol. Ecol. 13:563-571.

49. Zhao, X., Xue, C., Kim, Y., and Xu, J.-R. 2004. A ligation-PCR approach for generating gene replacement constructs in Magnaporthe grisea. Fungal Genet. Newsl. 51:17-18. 